\title{
Actualités en recherche en oncologie : I'essentiel du 4e Symposium International 2019 du Centre de Recherche en Cancérologie de Lyon
}

Frédéric Catez ${ }^{1}$, Nicole Dalla-Venezia ${ }^{1}$, Jean-Jacques Diaz ${ }^{1}$, Bertrand Dubois ${ }^{1}$, Anthony Ferrari ${ }^{2}$, Boris Guyot $^{1}$, Virginie Marcel ${ }^{1}$, Maria Ouzounova ${ }^{1}$, Roxane M Pommier ${ }^{2}$, Alain Viari ${ }^{2,3}$, Patrick Mehlen ${ }^{1}$

Tous les auteurs apporté une contribution équivalente.

${ }^{1}$ UnivLyon, Université Claude Bernard Lyon 1, INSERM 1052, CNRS 5286, Centre Léon-Bérard, Centre de Recherche en Cancérologie de Lyon, 28 rue Laennec, Lyon, France

${ }^{2}$ Synergie Lyon Cancer, Plateforme de bioinformatique Gilles Thomas, Centre Léon Bérard, 28 rue Laennec, Lyon, France

${ }^{3}$ Equipe Erable, INRIA Grenoble-Rhône-Alpes, 655 Avenue de l'Europe, 38330 Montbonnot-Saint Martin, France.

Le $4^{\mathrm{e}}$ Symposium International du CRCL (UMR Inserm 1052 - CNRS 5286 - UCBL - CLB) s'est déroulé au Centre de Congrès de Lyon du 2 au 4 octobre 2019 et a réuni plus de 550 participants. L'événement, qui a vocation à rassembler les chercheurs, cliniciens et industriels intéressés par les dernières avancées de la recherche en cancérologie, était organisé autour de quatre sessions plénières et accueillait pour la première fois le Symposium Gilles Thomas. Retour sur les communications les plus marquantes de cette édition.

Cellules souches et plasticité des cellules cancéreuses. Maria Ouzounova ${ }^{1}$

L'hétérogénéité intra-tumorale et la plasticité des cellules cancéreuses sont des aspects clés du développement tumoral, et sont responsables des rechutes et de l'échappement tumoral. L'hétérogénéité tumorale est le résultat d'événements génétiques, mais aussi de modifications épigénétiques, de facteurs environnementaux et métaboliques, ainsi que de l'activation de voies oncogéniques. La plasticité cellulaire permet l'acquisition de changements phénotypiques, fournissant ainsi des capacités de survie et d'adaptation aux cellules cancéreuses, et favorisant l'initiation et le développement tumoral. Dans cette session, des intervenants de référence du domaine ont discuté du rôle de la plasticité de la cellule cancéreuse et de l'hétérogénéité intra-tumorale dans l'adaptation des cellules cancéreuses à la pression sélective pendant l'initiation et la progression tumorale.

Le cancer du sein est un cancer très hétérogène, à la fois au niveau moléculaire et anatomopathologique. Pour comprendre cette hétérogénéité et identifier la cellule d'origine du cancer, il est important d'analyser en détail la hiérarchie épithéliale mammaire normale. Jane Visvader (The Walter and Eliza Hall Institute, Australie) a présenté l'utilisation du nouveau marqueur Tspan8 qui, de pair avec Lrg5, identifie une sous-population de cellules souches mammaires quiescentes, mais très sensibles aux stimuli. De plus, des analyses sur cellule unique ont révélé une complexité inattendue au sein des compartiments basal et luminal. Des analyses à différentes étapes du développement de la glande mammaire ont fourni des connaissances sur les premiers événements précurseurs de la mise en place de chaque lignage épithélial mammaire. Des études de suivi de lignage combinés, avec imagerie confocale 3D, tri cellulaire et séquençage de l'ARN ont permis la visualisation et l'identification de potentielles cellules d'origine du cancer. Les résultats ont montré la plasticité intrinsèque des cellules tumorales mammaires et suggèrent que la transition épithéliomésenchymateuse (TEM), est un événement fréquent et important in vivo. 
La TEM est l'un des programmes transcriptionnels à l'origine des cellules souches cancéreuses dans le cancer du sein. Max Wicha (University of Michigan, Etats-Unis) a discuté de l'existence d'états de cellules souches cancéreuses présentant une plasticité entre un état épithélial (E) et un état mésenchymateux (M). Les cellules souches cancéreuses (E) et $(M)$ sont caractérisées par l'utilisation de voies métaboliques distinctes et des différences de sensibilité à des inhibiteurs de la glycolyse et de l'oxydoréduction. La transition entre les états $(E)$ et $(M)$ est régie par une régulation étroite des niveaux de ROS et de $\mathrm{N}$-acétyl cystéine. Plus spécifiquement, l'inhibition des voies de la glycolyse et des réponses anti-oxydantes régule négativement la croissance tumorale, le potentiel d'initiation tumorale et la formation des métastases. Dans un deuxième temps, le Pr. Wicha a discuté de la vulnérabilité immunogénique des cellules souches cancéreuses et le développement de stratégies de vaccins anticellules souches cancéreuses, en utilisant des cellules dendritiques combinées avec des antigènes partagés par les CSC. En effet, les voies d'auto-renouvellement des cellules souches sont stimulées par les cellules myélö̈des suppressives (MDSC) par Notch et STAT3. D'autre part les CSC peuvent échapper à la surveillance immunitaire via une régulation positive des voies des cellules T inhibitrices et PDL-1. L'ensemble de ces résultats suggère un potentiel thérapeutique majeur des vulnérabilités métaboliques et immunologiques des CSC et leur utilisation en clinique.

La réponse thérapeutique dépendante de l'état épithélial par opposition à l'état mésenchymateux a été largement discutée lors de la conférence principale de la session Cellules souches et plasticité de la cellule cancéreuse, présentée par le Pr. Robert Weinberg (Whitehead Institute, États-Unis). Comme cela a été montré dans des modèles murins de cancer du sein, l'immunothérapie peut altérer significativement la croissance des tumeurs épithéliales mais n'a cependant aucun effet sur les tumeurs mésenchymateuses. Cela est dû principalement aux différents microenvironnements tumoraux présents dans ces deux types de tumeurs. Le développement de thérapies devrait être dicté en partie par la plasticité des cellules épithéliales et leur tendance à spontanément transiter vers un état plus mésenchymateux. Les résultats présentés suggèrent que des régulateurs épigénétiques imposant un état épithélial sont neutralisés pour induire les cellules vers un état mésenchymateux résistant aux thérapies. Afin de développer des thérapies anti-mésenchymateuses les études présentées suggèrent l'utilisation d'inducteurs de ferroptose, ciblant préférentiellement des cellules activant un programme de TEM.

L'ensemble de ces études a mis en évidence les avancées importantes obtenues récemment dans le domaine de la plasticité des cellules cancéreuses et ont présenté des stratégies originales et prometteuses de ciblage thérapeutique des cellules souches cancéreuses.

\section{Microenvironnement tumoral et immunité}

Boris Guyot ${ }^{1}$, Bertrand Dubois ${ }^{1}$

Les cellules tumorales établissent de multiples interactions avec leur microenvironnement, constitué de cellules mésenchymateuses, endothéliales et immunitaires, de molécules solubles et de matrice extracellulaire. II est désormais reconnu que cet écosystème complexe joue un rôle actif dans différents aspects du cancer tels que son initiation, sa progression et sa sensibilité ou sa résistance aux thérapies, Décrypter les interactions, souvent réciproques, entre ces différents acteurs, impliquant notamment les réponses immunitaires, l'inflammation, l'angiogenèse et le métabolisme, est une étape essentielle pour la compréhension de la biologie et de la dynamique du cancer et contribuera au développement de nouvelles innovations thérapeutiques. Cette session a couvert plusieurs aspects de ce domaine, allant de la régulation moléculaire de la réponse immunitaire antitumorale au ciblage thérapeutique de l'angiogenèse tumorale, à l'utilisation du microenvironnement tumoral pour la 
vaccination contre le cancer et au décryptage de l'hétérogénéité tumorale par séquençage d'ARN sur cellule unique.

Yenkel Grinberg-Bleyer (Centre de Recherche en Cancérologie de Lyon) nous a présenté ses travaux sur le rôle différentiel des sous-unités du facteur de transcription NF- $\kappa B$ dans la régulation de la réponse immunitaire antitumorale. Des expériences de délétion conditionnelle dans les cellules foxp ${ }^{+}$ montrent que c-Rel est essentiel pour le développement des lymphocytes T régulateurs (Tregs) dans le thymus, tandis que RelA est requis pour le maintien de leur identité en périphérie et de la tolérance immunitaire (Oh et al. Immunity 2017). De plus, c-Rel joue un rôle critique dans l'induction et le maintien de Tregs activés, souvent présents dans le microenvironnement tumoral, et la délétion de cette sous-unité NF-KB spécifiquement dans les Tregs ou son inhibition pharmacologique conduit à une diminution de la croissance tumorale, une meilleure efficacité de l'immunothérapie ciblant PD1 et une augmentation de la réponse $\mathrm{TCD}^{+}$(Grinberg-Bleyer et al. Cell 2017). Des résultats préliminaires montrent à l'inverse que p65 pourrait jouer un rôle critique dans la fonction des lymphocytes effecteurs en stimulant leur prolifération et la production de cytokines. L'ensemble de ces données suggère que le ciblage des sous-unités de NF-אB pourrait être utilisé pour stimuler la réponse antitumorale et augmenter l'efficacité des immunothérapies en modulant la fonction des lymphocytes $T$ effecteurs et/ou régulateurs.

Une autre stratégie pour augmenter l'efficacité des immunothérapies a été présentée par Michele De Palma (Ecole Polytechnique Fédérale de Lausanne, Suisse) : cibler l'angiogenèse tumorale, à l'origine de vaisseaux sanguins dysfonctionnels limitant l'extravasation des lymphocytes T. Le blocage simultané du VEGF et de l'angiopoiétine 2 à l'aide d'un anticorps bispécifique conduit à une régression vasculaire, à une normalisation des vaisseaux restants et à une régression tumorale dans des modèles murins de mélanome, de cancer du sein et du pancréas (Schmittnaegel et al. Sci. Transl. Med. 2017). Cet effet antitumoral est associé à une augmentation de la présentation d'antigènes tumoraux et du recrutement de lymphocytes $T C D 8^{+}$effecteurs, et peut être potentialisé par le blocage de PD1 ou l'ajout d'un agoniste de CD40 qui conduit à la reprogrammation des macrophages vers un profil M1.

Alexandra Lainé (Centre de Recherche contre le Cancer de Lyon) a montré que le TGF- $\beta$, un puissant suppresseur du système immunitaire très souvent secrété en grande quantité dans les tumeurs solides sous une forme inactive, est activé par l'intégrine $\beta 8$ (ITGB8) exprimée à la surface des Tregs Foxp3 $3^{+}$. La délétion spécifique d'ITGB8 dans les Tregs bloque la signalisation TGF- $\beta$ dans les lymphocytes T infiltrant les tumeurs et permet une réponse cytotoxique anti-tumorale efficace. Ces résultats éclairent les rôles respectifs des cellules tumorales et des Tregs lors de la suppression de la réponse immune anti-tumorale via la sécrétion et l'activation du TGF- $\beta$.

Itay Tirosh (Weizmann Institute of Science, Israël) a présenté des résultats d'analyses transcriptomiques par RNA-seq sur cellule unique d'échantillons de plusieurs types de tumeurs avec une insistance particulière sur les cancers des voies aérodigestives supérieures. Ces données transcriptomiques ont permis d'identifier des sous-populations intra-tumorales récurrentes d'intérêt clinique, comme par exemple des populations présentant des caractéristiques de cellules souches, de cellules invasives ou de cellules résistantes aux traitements. Le conférencier a souligné l'intérêt et les difficultés d'identifier ces différentes signatures moléculaires dans les cellules malignes et de les isoler des signatures issues de cellules stromales dans les données transcriptomiques générées à partir d'échantillons tumoraux hétérogènes.

Une contribution à l'étude multidisciplinaire MESOMICS sur le mesothéliome pleural malin (MPM), un cancer rare mais très agressif causé par l'exposition aux fibres d'amiante, a été exposée par Lise Mangiante (Centre International de Recherche sur le Cancer, Lyon). Des analyses transcriptomiques par RNA-seq d'échantillons tumoraux issus du réseau national multicentrique MESOBANK ont permis 
de corréler le pronostic des patients avec des profils moléculaires distincts. Les groupes de pronostics extrêmes sont caractérisés par une expression différentielle de gènes impliqués dans les check-points immunitaires et dans l'angiogenèse. De plus, des réarrangements génomiques récurrents affectant le gène suppresseur de tumeurs $B A P 1$, qui était déjà connu pour être fréquemment muté dans les MPM, ont été identifiés. La découverte de profils moléculaires distincts, corrélés avec le pronostic des patients et impliquant le contrôle de la réponse immunitaire et l'angiogenèse sont importants pour l'élaboration de futures stratégies thérapeutiques visant le MPM.

Les plus-values de l'immunothérapie intra-tumorale, tant pour ses effets secondaires limités que pour sa capacité à contourner des résistances aux thérapies ciblant PD1/PD-L1 et CTLA4, ont été exposées par Aurélien Marabelle (Institut Gustave-Roussy, Villejuif). Ses travaux très récents démontrent ainsi que des vaccins anti-infectieux déjà sur le marché peuvent être utilisés comme source de ligands de récepteurs de l'immunité innée pour induire ou stimuler une réponse immunitaire anti-tumorale. II montre, dans des modèles murins de neuroblastome et de lymphome, que l'injection intra-tumorale de vaccins anti-rotavirus a un effet oncolytique, induit une régression tumorale et une réponse abscopale par un mécanisme impliquant les lymphocytes $\mathrm{T} \mathrm{CD4}^{+}, \mathrm{CD}^{+}$et $\mathrm{NK}$, et permet de lever la résistance aux inhibiteurs de PD1 et CTLA4 (Shekarian et al. Sci. Transl. Med. in press).

\section{Ribosome, traduction et cancer.}

\section{Frédéric Catez ${ }^{1}$, Virginie Marcel $^{1}$, Nicole Dalla Venezia ${ }^{1}$, Jean-Jacques Diaz ${ }^{1}$}

Avec la régulation transcriptionnelle et épigénétique, la régulation de la traduction joue un rôle capital dans I'homéostasie et l'identité cellulaire. Néanmoins, ce mode de régulation de l'expression génique reste encore peu exploré en cancérologie, et ce malgré un rôle central déjà démontré dans l'établissement et la progression du phénotype tumoral. Ces dernières années, plusieurs découvertes majeures ont marqué ce domaine. C'est ainsi que les altérations de la machinerie de traduction (ribosomes, ARN de transfert et facteurs de traduction) ont émergé comme une nouvelle " caractéristique " des cellules cancéreuses, caractéristique qui apporte aux cellules un niveau supplémentaire de régulation de l'expression génique permettant d'acquérir des propriétés proprolifératives et de plasticité accrues.

La variabilité d'expression des gènes codant les protéines ribosomiques et l'existence de gènes paralogues codant des protéines ribosomiques a conduit à l'hypothèse que toute cellule est capable de produire non pas, un type de ribosomes, mais plusieurs types de ribosomes, avec des conséquences possibles sur la synthèse de certaines protéines. Cette hypothèse n'avait pas été directement démontrée jusqu'à récemment. Maria Barna (Stanford University, États-Unis) a rapporté la caractérisation de ribosomes dont le contenu en protéines ribosomiques varie (telles que RPL38, RPS25, RPL10A, RPL40). Une approche de purification spécifique de ces ribosomes de composition variable révèle que ces ribosomes s'associent de façon différentielle aux ARNm. Ce travail, réalisé dans des cellules embryonnaires murines, a montré que les protéines RPS25 et RPL10A contribuent à la régulation de la traduction d'ARNm codant des protéines impliquées dans des voies importantes en cancérologie telles que la matrice extracellulaire, la croissance cellulaire, la réponse au stress et le cycle cellulaire. Un premier mécanisme a été proposé, mettant en jeu des éléments IRES (Internal Ribosome Entry Sites) présents dans la région 5' non traduite des ARNm.

Au sein du ribosome, les ARN ribosomiques (ARNr) sont les porteurs de l'activité de décodage de I'ARNm et d'assemblage des acides-aminés en protéine. La présence de plus de 200 modifications chimiques sur les ARNr est une source potentielle de variabilité du ribosome, encore très peu explorée. Grace à une technologie innovante basée sur le RNA-Seq, appelée RiboMethSeq, Jean-Jacques Diaz (Centre de Recherche en Cancérologie de Lyon) a rapporté que la 2'-O-méthylation des ARNr varie 
dans des modèles d'initiation tumorale, alors que cette modification était jusque-là considérée comme constitutive. Cette variabilité s'accompagne d'une modulation de la traduction IRES-dépendante d'ARNm de gènes clefs de la tumorigenèse, tels que les gènes IGF1R, MYC ou les récepteurs du FGF1 ou du VEGFA. Une première étude sur une cohorte de 200 tumeurs mammaires a révélé que la méthylation de certains nucléotides est particulièrement altérée, montrant pour la première fois l'hétérogénéité de l'ARNr chez les patientes atteintes d'un cancer du sein. Ces données ouvrent de nouvelles perspectives tant pour l'identification de biomarqueurs que de cibles thérapeutiques.

La contribution des grandes voies oncogéniques et suppressives dans l'établissement de programmes de traduction spécifique des cellules cancéreuses est une question majeure, explorée depuis quelques années par Davide Ruggero (University of California at San Francisco, Etats-Unis). Il a rapporté le rôle de l'oncogène $M Y C$ dans un contexte RAS ${ }^{G 12 D}$ dans un modèle de cancer hépatique chez la souris. Tandis que la synthèse protéique globale n'est pas modifiée, la traduction des ARNm codés par des gènes impliqués dans la réponse immunitaire est sélectivement modulée. En particulier, la surexpression de $M Y C$ stimule la synthèse de la protéine PD-L1, en contournant l'utilisation d'un cadre de lecture précoce (uORF) présent dans I'ARNm de PD-L1. En conséquence, l'augmentation de PD-L1 dépendante de MYC réduit la réponse immunitaire intra-tumorale et augmente le potentiel métastatique de la tumeur primaire.

L'altération de la machinerie de traduction et de la synthèse protéique ont ainsi émergé comme des critères distinctifs des cellules cancéreuses vis-à-vis des cellules saines. La possibilité de cibler la machinerie de traduction est soutenue par plusieurs études majeures qui ont apporté la preuve du concept. Cela comprend notamment des inhibiteurs de la biogenèse des ribosomes (anti-RNA polymérase I) et du complexe d'initiation de la traduction (anti-eIF4F). Dans ce contexte, Jean-François Peyron (Centre Méditerranéen de Médecine Moléculaire, Nice) a présenté des données sur le développement d'inhibiteurs de la synthèse protéique permettant de cibler des leucémies associées à une sur-activation de l'oncogène Myc. En effet, l'équipe de Jean-François Peyron a montré l'addiction des cellules de leucémie lymphoïde aiguë à cellules T (T-ALL) à certains acides aminés et à la synthèse protéique. Des résultats très prometteurs sur plusieurs composés ont été présentés.

Ces études viennent enrichir un champ émergent de la biologie du cancer qui place la machinerie de traduction, et plus particulièrement le ribosome, ses protéines et les modifications chimiques de ses $\mathrm{ARN}$, comme des acteurs clés de la régulation traductionnelle et de la tumorigenèse. Ces données permettent de mieux comprendre les processus d'initiation et de progression tumorale (dont la formation de métastases), et ont ouvert des voies innovantes et inattendues pour l'identification de nouveaux biomarqueurs et de nouvelles cibles thérapeutiques.

\section{Symposium Gilles Thomas - Cancer Genetics}

Roxane M Pommier ${ }^{2}$, Anthony Ferrari ${ }^{2}$, Alain viari ${ }^{2,3}$

Le $3^{\mathrm{e}}$ symposium Gilles Thomas s'est déroulé le vendredi 04 octobre 2019 à Lyon, conjointement à la session « Cancer Genetics » du $4^{\mathrm{e}}$ symposium du CRCL. II fait suite aux précédentes éditions qui se sont tenues à l'Institut Pasteur en janvier 2015 puis à I'Université Paris-Descartes en octobre 2017. Ces symposiums, dédiés à la mémoire du Pr Gilles Thomas, qui nous a quitté en 2014, ont été initiés dans la communauté de la génomique des cancers par des collègues qui ont connu Gilles et travaillé avec lui, en particulier Jessica Zucman-Rossi, Anne Vincent-Salomon, Sylviane Olschwang, Olivier Delattre, Pierre Laurent-Puig, Alain Puisieux et Fabien Calvo.

En ouverture de la journée, Alain Viari, directeur scientifique de la plate-forme bioinformatique « Gilles Thomas ", a rappelé le contexte de l'installation de Gilles Thomas à Lyon en 2008. II a notamment 
exposé les rôles déterminants qu'ont tenus, sur le plan organisationnel, l'Institut National du Cancer et la Fondation Synergie-Lyon-Cancer et, sur le plan scientifique, le programme « International Cancer Genome Consortium " (ICGC) dans la structuration de la plateforme de bioinformatique. En miroir de cette introduction, Anthony Ferrari a présenté, en fin de journée, un bilan de la participation de la France dans I'ICGC avec un accent particulier sur le programme " cancers du sein HER2-amplifiés " dont Gilles était le responsable scientifique.

Les exposés, de très grande qualité, ont été centrés sur trois thèmes majeurs.

\section{Processus oncogénique et les signatures mutationnelles.}

Serena Nik-Zainal (Université de Cambridge, Royaume-Uni) a présenté un riche et enthousiasmant panorama de ses travaux sur les traces génomiques (signatures mutationnelles et structurales) des processus oncogéniques, notamment sur les cancers du sein dans le cadre de I'ICGC. Quentin Bayard (Centre de Recherche des Cordeliers, Paris) a présenté une mise en œuvre très convaincante de ces mêmes approches dans le cas des hépatocarcinomes. Avec un prisme plus épidémiologique, Paul Brennan (Centre International de Recherche en Cancérologie, Lyon) a présenté Mutograph, un projet pan-cancer de Cancer Research UK visant à caractériser les signatures mutationnelles des cancers de plus de 5000 individus repartis sur 20 pays des cinq continents. Enfin, d'un point de vue plus translationnel, Yves Pommier, du National Cancer Institute, États-Unis, a détaillé l'intérêt des lignées cellulaires en culture dans la prédiction de réponse aux traitements en l'illustrant avec le cas de la protéine SLFN11 et les thérapies ciblant la réplication de l'ADN.

\section{Évolution tumorale}

La modélisation de l'oncogenèse, vue comme un processus d'évolution à l'échelle multicellulaire, est devenue un sujet de fort intérêt sur le plan international. Cela a été illustré brillamment par deux exposés consécutifs : Trevor Graham (Barts Cancer Institute, Royaume-Uni) a présenté les concepts et modèles mathématiques de la dynamique d'évolution des sous-clones tumoraux. Pierre Martinez (Centre de recherche en Cancérologie de Lyon) a présenté une approche basée sur l'observation des co-occurences d'altérations génomiques pour modéliser l'initiation et la progression tumorale ainsi que l'adaptation des sous-clones à l'environnement cellulaire.

\section{Hétérogénéité tumorale}

Le problème de l'hétérogénéité intra-tumorale est une question centrale pour la compréhension de l'oncogenèse mais aussi pour la prise en charge thérapeutique. Dans ce domaine, Nicolas Alcala (Centre International de Recherche sur le cancer, Lyon) a présenté une élégante approche multiomique, alliant génome, transcriptome et méthylome, permettant d'appréhender l'évolution temporelle et spatiale des tumeurs, illustrée par le cas des tumeurs thoraciques. La question de la plasticité tumorale, notamment lors du changement d'identité cellulaire (trans/dé/différentiation), a été remarquablement illustrée par Isabelle Janoueix-Lerosey (Institut Curie, Paris) sur l'exemple du neuroblastome. Enfin, cette question est, depuis quelques années, abordée avec succès par les approches à l'échelle de la cellule unique, ainsi que Manfred Claassen (ETH Zurich, Suisse) l'a brillamment exposé.

Enfin, la thématique fondamentale de l'épigénétique a également été abordée par Edith Heard (EMBL, Allemagne) dans une présentation exhaustive sur l'inactivation du chromosome $X$. La journée a été clôturée par une présentation du Dr Mehmet Oztürk (Izmir Genome Center, Turquie) qui a présenté un récapitulatif très personnel de son admirable carrière scientifique, ayant notamment donné lieu aux prémices de l'actuel CRCL. 